\title{
Ionic Polymer-Metal Composite Electro-Active Nano-biopolymer Actuator
}

\author{
Ravi Kumar Cheedarala and Jung Il Song \\ Research Institute of Mechatronics, Department of Mechanical Engineering, Changwon National University, Uichang-gu, \\ Changwon City, Gyeongnam, South Korea
}

\begin{abstract}
This research article introduces an electroactive actuator based on sulfonated chitosan (SCS), ionic liquid and graphene oxide (GO). To maintain the biocompatibility of the chitosan and to increase the actuation performance, a biocompatible ionic liquid such as ethyl, methylimidazolium-trifluoro methane sulfonated (EMI-TFMS) as mobile solvent was used for dry-type actuation. And GO-reinforced SCS films obtained through a simple solvent casting method were extensively investigated because of better electro-chemo-mechanical properties and higher actuation performances. Field-emission scanning electron microscope (FE-SEM), X-ray diffraction (XRD), Fourier transformer infrared spectroscopy (FT-IR), and Raman spectra were used to investigate the interaction mechanism between GO and SCS. The harmonic and step responses of GO and SCS composite actuator show large bending deformations under low electric voltage.
\end{abstract}

Key words: Chitosan, ionic liquid, GO, bio-mimetic actuator, nano-biopolymer.

\section{Introduction}

Development of economic, flexible, lightweight and eco-friendly biocompatible ionic polymer-metal composite (IPMC) actuators is a thought-provoking task in electro-chemo-mechanical actuator materials (ECMA). ECMAs are directly converting electrical energy into mechanical energy and they display huge potential applications including artificial muscles for robots, biomimetic flying objects, optical switches, and microsensors, etc. [1]. Since many years, ECMAs have been made from conductive polymers, carbon nanotubes, graphene oxide (GO), dielectric elastomers and ferroelectric polymers [2]. Among them, ferroelectric polymers and dielectric elastomers are performing large strain, fast stimuli response, and strong electromechanical coupling, but they generally require high driving voltages and high activation fields which limit their potential applications [3]. To overcome this issue, we wanted to develop a novel biocompatible ionic liquid-GO-reinforced-chitosan for ECMAs [4].

Corresponding author: Jung Il Song, Ph.D., professor, research field: natural fiber composits.
Ionic liquids (ILs) are organic liquids composed of ions that are colorless fluids and easy to handle. Importantly, low vapor pressure, excellent tunable solubility, and high thermal stability of ILs have attracted much attention as green solvents [5]. Chitosan (CS) displays excellent film-forming property with less flexibility due to inferior mechanical and chemical structure [6]. To increase its mechanical strength, GO is one of the alternatives as a filler to mix well with CS due to the formation of strong hydrogen bonding between the carboxyl group (COOH) of $\mathrm{GO}$ and amine $\left(\mathrm{NH}_{2}\right)$ group of CS. Employing SCS as a mediating agent for GO dispersing may bring about an interesting system that is SCS, Scheme 1.

\section{Experimental}

\subsection{Synthesis of SCS-GO Films}

GO (0.10-0.30 g) was dissolved in $100 \mathrm{~mL}$ of deionized (DI) water and applied by mild ultra-sonication for converting into a homogeneous suspension. Next, $1 \mathrm{~mL}$ acetic acid (AcOH) and $1.5 \mathrm{~g}$ sulfonated chitosan (SCS) were added sequentially into 


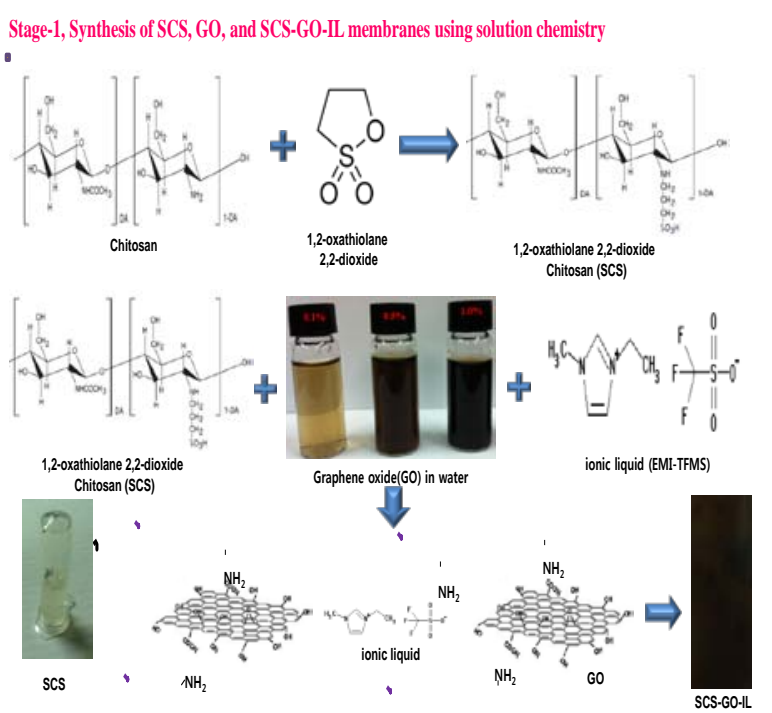

Stage-2, Preparation of SCS-GO-IL actuator and Sinusoidal actuation phenomenon at applied voltages.

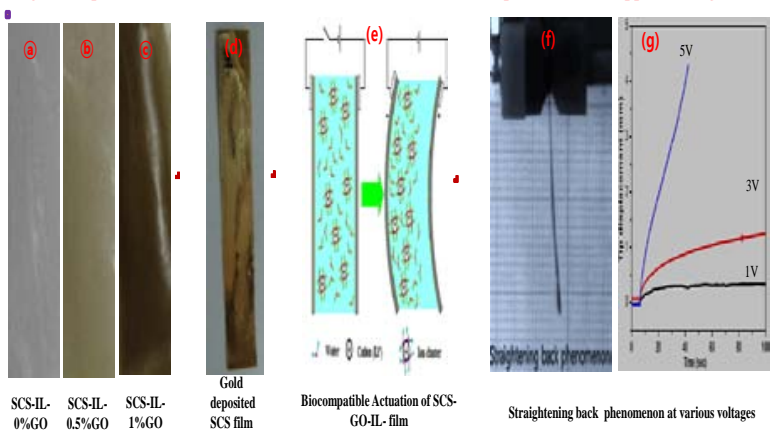

Fig. 1 Schematic illustration of SCS-GO-IL membranes for robust bio-mimetic actuation at various applied voltages.

the GO suspension while stirring. After 2 h, SCS-GO solution mixture was transferred into a plain flat glass plate to get bio-nano films with various wt $\%$ of GO (i.e. $0.1 \mathrm{wt} \%, 0.5 \mathrm{wt} \%$, and $1.0 \mathrm{wt} \%)$, as shown in Fig. 1. Finally, the composite films were dried at $40{ }^{\circ} \mathrm{C}$ in a hot air oven for further experiment. By changing the weight percent's of SCS to GO, such as 1.5:0.1, 1.5:0.5, and 1.5:1.0, a series of SCS-GO composite films were obtained with the codes of SCS-GO-1, SCS-GO-2, and SCS-GO-3. The obtained composite films were soaked in EMI-TFSA IL for $24 \mathrm{~h}$ to be ready for the actuation performance.

\subsubsection{Electrochemical Applications}

SCS-GO nano-biopolymer was determined using ion exchange capacity (IEC), ionic conductivity (IC), and electrochemical impedance spectroscopy (EIS) analysis. The number of $\mathrm{m}$. eq. of ions in 1,000 $\mathrm{mg}$ of the dry SCS-GO nano-biopolymer was analyzed by IEC. The proton $\left(\mathrm{H}^{+}\right)$form of the film was transformed into the $\mathrm{Na}^{+}$ion form by dipping in $1 \mathrm{M} \mathrm{NaCl}$ solution for $24 \mathrm{~h}$. The exchanged ions in the solution were titrated against $0.1 \mathrm{M} \quad \mathrm{NaOH}$ solution and phenolphthalein as an indicator. The IEC values were determined by the following equation:

$I E C=\frac{\text { Consumed } \mathrm{NaOH}(\mathrm{mL}) \times \text { Molarity of } \mathrm{NaOH}}{\text { Weight of the Membrane }}$

The ionic exchange capacity of the EMI-TFSA IL doped films was valued by using the EIS from a complex impedance analyzer over a frequency range of 10-1 MHz under a maximum applied voltage of 0.1 V. Films of a circular shape with a $6 \mathrm{~mm}$ in diameter and an ECC-STD electrochemical cell with two steel electrodes were used. The IC can be determined by the following equation:

$$
\text { Ionic Conductivity }=\frac{1}{R(\Omega)} \times \frac{L(\mathrm{~cm})}{S\left(\mathrm{~cm}^{2}\right)}[\mathrm{s} / \mathrm{cm}]
$$

where $R$ is the ionic resistance, which is calculated from the intercept of the semicircle at the low-frequency side of the Nyquist plot with the real axis, and $L$ and $S$ are the thickness and area of the films. The obtained research data by EIS were fitted by using ZSimpWin 3.21 software.

\subsection{The Fabrication Process of SCS-GO and Its Set-Up for Actuation Tests}

Thin gold $\mathrm{Au}$ ) electrodes were deposited on both sides of the as-received SCS-GO films using an Au spin coater. The size of the actuator was $4 \mathrm{~mm} \times$ $20 \mathrm{~mm} \times 0.3 \mathrm{~mm}$. The SCS-GO actuator was clamped with about a $4 \mathrm{~mm} \times 5 \mathrm{~mm}$ area at one end. The experimental setup for the measurement of actuation consisted of a PXI 6252 data acquisition board, a current amplifier (UPM1503, Quanser), a charged-couple device camera (XC-HR50, Sony) and a laser displacement sensor (LK-031, Keyence). All the data were received as such and controlled by an NI-PXI system (1042Q, NI) by using the LabVIEW program. 
The bending curvature $\kappa$ generated in the actuator was estimated by the following equation.

$$
K=\frac{1}{R}=2 \delta /\left(I^{2}+\delta^{2}\right)
$$

where $R, \delta$, and $l$ are the radius of curvature, the tip displacement and the free length of the actuator.

\section{Results and Discussion}

\subsection{Scanning Electron Microscope (SEM)}

Fig. 2 shows SEM images of SCS-IL-GO nanocomposites with different GO loadings (0.1 wt\%, $0.5 \mathrm{wt} \%$, and $1 \mathrm{wt} \%)$. From images of film surfaces, no obvious difference between pure SCS-IL and SCS-IL-GO nanocomposites can be observed. It shows the well-dispersed status of SCS-IL and GO without any agglomeration because of self-assembled layer-by-layer network. Wrinkled patches/folds on the surface were found due to the increment of GO nanoflakes loading on the SCS-IL membrane (Figs. 2b-2d).

\subsection{FT-IR, XRD, and Raman Spectra}

Fig. 3a shows the FT-IR spectra SCS-IL and GO and their chemical interactions. The peaks at $1,718 \mathrm{~cm}^{-1}$ and $1,630 \mathrm{~cm}^{-1}$ are representing the $\mathrm{C}=\mathrm{O}$ (str) of $\mathrm{COOH}$ on the $\mathrm{GO}$ and (deform) of the $\mathrm{OH}$ bond in

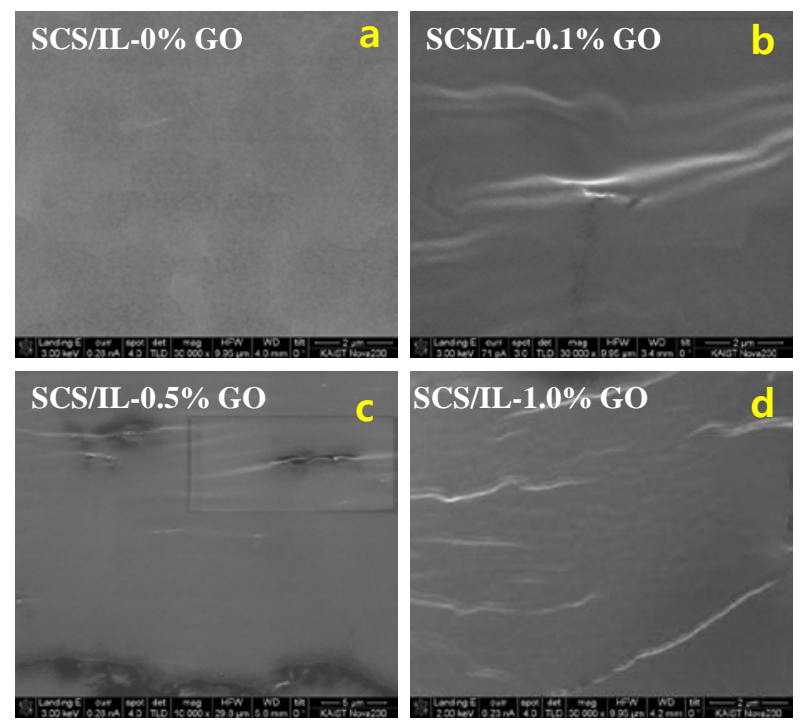

Fig. 2 Surface SEM images of SCS-IL-GO membranes.

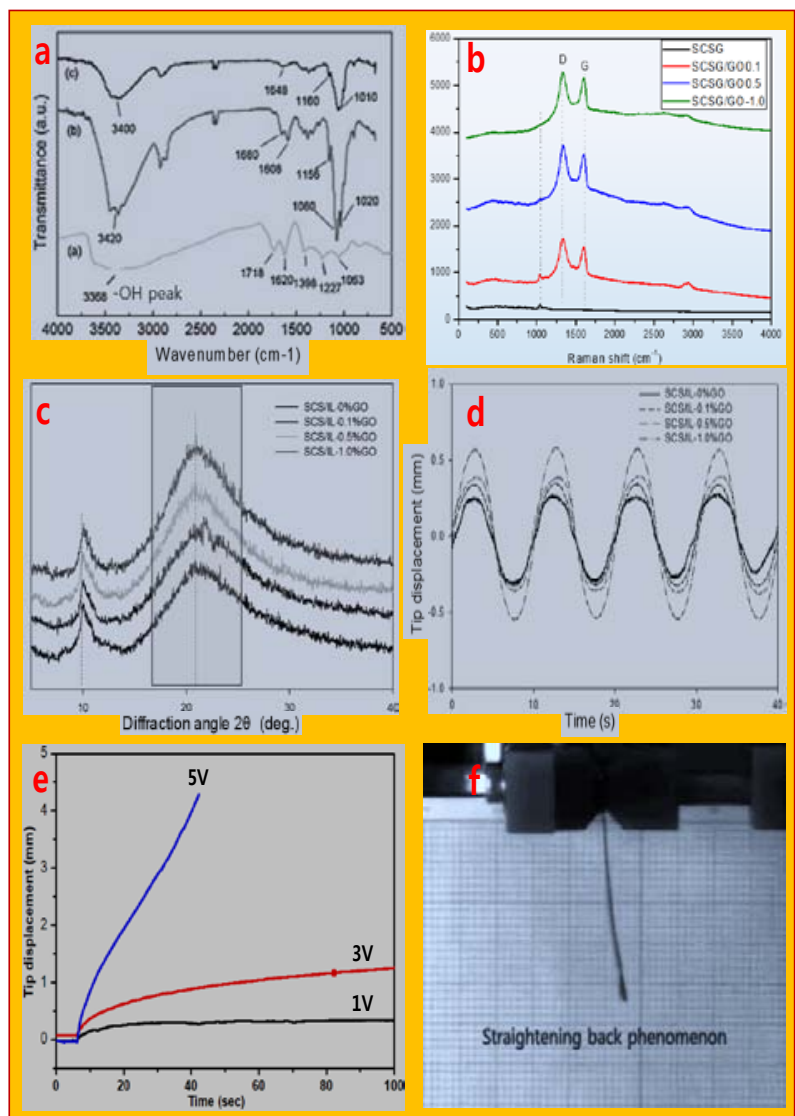

Fig. 3 Characterizations of SCS-IL-GO actuator. (a) FT-IR, (b) Raman, (c) XRD, (d) harmonic response of sinusoidal wave, (e) step responses and (f) straightening back phenomenon at applied voltages.

water, respectively. In the spectrum of SCS-IL-GO, mainly, two peaks obtained at $1,652 \mathrm{~cm}^{-1}$ and 1,561 $\mathrm{cm}^{-1}$, correspond to the $\mathrm{C}=\mathrm{O}$ peak of amide (-NH-CO-) and $\mathrm{NH}$ bending peak of $-\mathrm{NH}_{2}$, respectively [7]. The broad absorption peaks appeared at $\sim 2,900 \mathrm{~cm}^{-1}$ and $3,300-3,400 \mathrm{~cm}^{-1}$ which represents $\mathrm{SO}_{3} \mathrm{H}$ and $\mathrm{OH}$ groups present on SCS-IL and GO.

Raman spectra of GO display two normal bands at 1,333 $\mathrm{cm}^{-1}$, and $1,580 \mathrm{~cm}^{-1}$ for the disorder-induced D-band and G-band, and a shoulder around $1,620 \mathrm{~cm}^{-1}$ assigned to the D' band, as shown in Fig. 3b [7]. Also, the stretching vibration is at $1,037 \mathrm{~cm}^{-1}$ for S-O-S in SCS-IL. However, it is noticed that the intensity of characteristic GO bands increases with the increment of loading of GO from $0.1 \mathrm{wt} \%, 0.5 \mathrm{wt} \%$ and $1.0 \mathrm{wt} \%$ in the SCS-IL membrane matrix. It is strongly suggested a strong interaction between SCS-IL and the GO. 
XRD analysis reveals that the GO sheets present in the SCS-IL-GO nanocomposite membranes, as shown in Fig. 3c. A broad peak and a sharp peak at $2 \theta$ value of $20.3^{\circ}$ and $10^{\circ}$, which are clear evidence of the amorphous nature of SCS film and GO, respectively. However, it is noticed that the intensity of characteristic peaks of SCS-IL-GO was increased with the loading of GO sheets from $0.1 \mathrm{wt} \%, 0.5 \mathrm{wt} \%$ and $1 \mathrm{wt} \%$ in the SCS-IL matrix and also increased the peak heights [8-12].

\subsection{Harmonic Responses of SCS-IL-GO Actuator}

Fig. 3d shows the harmonic motion of the pure SCS-IL (0 wt $\%$ ) of GO, SCS-IL-0.1 wt $\%$ GO, SCS-IL-0.5 wt\% GO and SCS-IL-1.0 wt\% GO actuators under an applied voltage of AC $1 \mathrm{~V}$ with an excitation frequency of $0.1 \mathrm{~Hz}$. The tips displacement of the SCS-IL-GO actuator, which had the highest weight ratio of GO (1.0 wt\%) was (SCS-IL-1\% GO) $0.57 \mathrm{~cm}$, which was 2.28 times more than SCS/IL with the tip displacement of $0.025 \mathrm{~cm}$ under the input voltage of $2 \mathrm{~V}$. The tip displacements were $0.4 \mathrm{~cm}$ for SCS-IL $0.5 \mathrm{wt} \% \mathrm{GO}$ and $0.342 \mathrm{~cm}$ for SCS-IL $0.1 \mathrm{wt} \%$ GO, respectively [9, 10]. Next, we also study selectively the actuation performance with SCS-IL-0.1 wt\% GO actuator at the AC voltages from 2 to $10 \mathrm{~V}$ at the same excitation frequency $[11,12]$. The displacement of the SCS/IL-0.1 wt\% GO actuators was $10.5 \mathrm{~mm}$ from an applied voltage at $10 \mathrm{~V}, 8.7 \mathrm{~mm}$ from at $5 \mathrm{~V}$, $3.2 \mathrm{~mm}$ from $3 \mathrm{~V}$ and $2.1 \mathrm{~mm}$ from $2 \mathrm{~V}$ with an excitation frequency of $0.1 \mathrm{~Hz}$, respectively, which indicate that these nanocomposites actuators are promising candidates for future high-performance actuators (Figs. 3e and 3f) [13-19]. In particular, the highest tip displacement was shown by SCS-IL-1.0 wt\% GO due to strong ion-ion interactions between $\mathrm{SO}_{3} \mathrm{H}$ and $\mathrm{COOH}$ and IL during actuation performance of various applied voltages of 2-10 V.

\section{Conclusions}

In summary, we demonstrate novel SCS-IL-GO based bio-nano actuators that have biocompatible and biodegradable properties and are newly developed as an electro-active biopolymer. High-performing nano-bio-polymer actuator shows a large bending deformation under low electrical field in open-air condition due to the functionalities such as sulfonated chitosan, doping of biocompatible ionic liquid and reinforcing of GO. The bending deformation improvement was obtained due to the higher ionic conductivity, larger capacitance, and delay of ionic liquid migration in the reduced ionic pores or channels of GO (1.0 wt\%) SCS-IL under lower applied voltages. Surprisingly, the curvature of the actuator under the 3 $\mathrm{V}$ step input was enhanced as much higher as we can not determine the tip displacement with laser displacement sensors. Present results showed that the biocompatible chitosan-based bio-nano actuator can be a promising smart material and may have diverse applications for the active-biomedical devices which are required for the next generation smart electronic products such as wearable soft electronics, flexible displays, and smart mobile phones.

\section{Acknowledgments}

National Research Foundation of Korea (NRF) No. 2017R1A2B2011730, No. 2018R1A6A1A03024509 and 2019R1A2C1011113.

\section{References}

[1] Mohsen, S., and Kwang, J. K. 2001. “Ionic Polymer-Metal Composites: I. Fundamentals.” Smart Mater. Struct. 10: 819-33.

[2] Rajagopalan, M., and Oh, I. K. 2011. "Fullerenol-Based Electroactive Artificial Muscles Utilizing Biocompatible Polyetherimide.” ACS Nano 5: 2248-56.

[3] Baughman, R. H., Cui, C., Zakhidov, A. A., Iqbal, Z., Barisci, J. N., Spinks, G. M., et al. 1999. "Carbon Nanotube Actuators.” Science 284: 1340-4.

[4] Cheedarala, R. K., Jeon, J. H., Key, C. D., and Oh, I. K. 2014. "Bio-Inspired All-Organic Soft Actuator Based on a $\pi-\pi$ Stacked 3D Ionic Network Membrane and Ultra-Fast Solution Processing.” Adv. Funct. Mater. 24: 6005-15.

[5] Fukushima, T., Asaka, K., Kosaka, A., and Aida, T. 2005. "Fully Plastic Actuator through Layer-by-Layer Casting with Ionic-Liquid-Based Bucky Gel.” Angew Chem Int 
Ed Engl 44: 2410-3.

[6] Li, J., Ma, W., Song, L., Niu, Z., Cai, L., Zeng, Q., and Zhang, X., et al. 2011. "Superfast-Response and Ultrahigh-Power-Density Electromechanical Actuators Based on Hierarchal Carbon Nanotube Electrodes and Chitosan.” Nano Lett. 11: 4636-41.

[7] Fennimore, A. M., Yuzvinsky, T. D., Han, W. Q., Fuhrer, M. S., Cumings, J., and Zettl, A. 2003. "Rotational Actuators Based on Carbon Nanotubes.” Nature 424: 408-10.

[8] Lehmann, W., Skupin, H., Tolksdorf, C., Gebhard, E., Zentel, R., Kruger, P., Losche, M., and Kremer, F. 2001. "Giant Lateral Electrostriction in Ferroelectric Liquid-Crystalline Elastomers.” Nature 410: 447-50.

[9] Lu, W., Fadeev, A. G., Qi, B., Smela, E., Mattes, B. R., Ding, J., et al. 2002. "Use of Ionic Liquids for $\pi$-Conjugated Polymer Electrochemical Devices.” Science 297 (5583): 983-7.

[10] Nguyen, V., Kim, J., Tabassian, R., Kotal, M., Jun, K., Oh, J. H., et al. 2019. "Electroactive Artificial Muscles Based on Functionally Antagonistic Core-Shell Polymer Electrolyte Derived from PS- $b$-PSS Block Copolymer.” Adv. Science 6: 1801196.

[11] Cheedala, R. K., Kim, G. H., Cho, S., Lee, J. H., Kim, J., Song, H. K., Kim, J. Y., and Yang, C. 2011. "Ladder-Type Heteroacenepolymers Bearing Carbazole and Thiophene Ring Units and Their Use in Field-Effect Transistors and Photovoltaic Cells.” J. Mater. Chem. 21: 843-50.

[12] Cheedarala, R. K., Park, E. J., Kong, K., Park, Y. B., and Park, H. W. 2016. "Experimental Study on Critical Heat Flux of Highly Efficient Soft Hydrophilic CuO-Chitosan Nanofluid Templates.” Int. J. Heat Mass
Trasfer 100: 396-406.

[13] Cheedarala, R. K., and Duy, L. C. 2018. "Double Characteristic BNO-SPI-TENGs for Robust Contact Electrification by Vertical Contact Separation Mode through Ion and Electron Charge Transfer." Nano Energy 44: 430-7.

[14] Cheedarala, R. K., and Parvez, A. N. 2018. "Electric Impulse Spring-Assisted Contact Separation Mode Triboelectric Nanogenerator Fabricated from Polyaniline Emeraldine Salt and Woven Carbon Fibers.” Nano Energy 53: 362-72.

[15] Cheedarala, R. K., Shahriar, M., Ahn, J. H., Hwang, J. Y., and Ahn, K. K. 2019. "Harvesting Liquid Stream Energy from Unsteady Peristaltic Flow Induced Pulsatile Flow-TENG (PF-TENG) Using Slipping Polymeric Surface Inside Elastomeric Tubing.” Nano Energy 65: 104017.

[16] Roy, S., Kim, J. W., Kotal, M., Tabassian, R., Kim, K. J., and Oh, I. K. 2019. "Collectively Exhaustive Electrodes Based on Covalent Organic Framework and Antagonistic Co-Doping for Electroactive Ionic Artificial Muscles.” Adv. Funct. Mater. 29: 1900161.

[17] Kotal, M., Kim, J., Tabassian, R., Roy, S., Nguyen, V. H., Koratkar, N., and Oh, I. K. 2018. "Highly Bendable Ionic Soft Actuator Based on Nitrogen-Enriched 3D Hetero-Nanostructure Electrode.” Adv. Funct. Mater. 28: 1802464.

[18] Kim, J. E., Oh, J. H., Kotal, M., Koratkar, N., and Oh, I. K. 2017. Nano Today 14: 100-23.

[19] Cheedarala, R. K., and Song, J. L. 2019. RSC Advances, InPress. 